\title{
ABSENCE OF ABSOLUTELY CONTINUOUS SPECTRA FOR MULTIDIMENSIONAL SCHRÖDINGER OPERATORS WITH HIGH BARRIERS
}

\author{
IVOR MCGILLIVRAY, PETER STOLLMANN AND GÜNTER STOLZ
}

\begin{abstract}
We prove absence of absolutely continuous spectra for multidimensional Schrödinger operators with high barriers. The result is formulated in terms of a geometric condition on the barriers which entails singular spectrum. The proof combines probabilistic and functional analytic techniques.
\end{abstract}

\section{Introduction}

In [10], Simon and Spencer exhibited two typical situations in which Schrödinger operators have singular spectrum, namely when their potential has 'wide barriers' or 'high barriers'. For the case of wide barriers, their results were formulated for discrete Schrödinger operators. Similar results for continuum operators were proven in [13] for dimension $d=1$, in [1] and [12] for arbitrary dimension; [13, 12] contain somewhat more refined results in terms of a comparison criterion, allowing one to prove absence of absolute continuity also at high energies. In this note we treat multidimensional Schrödinger operators with high barriers, thus extending the $d=1$ results of $[\mathbf{1 0}, \mathbf{4}, \mathbf{1 4}]$. While our general method of proof is that of [10], there are some differences which we would like to point out. Since adding a Dirichlet boundary condition is no longer a finite rank perturbation in $d \geqslant 2$, the necessary trace class estimates become more involved. Our key to establishing such estimates (apart from a factorization technique introduced in [11] and developed in [12]) is Lemma 3 below, which deals with occupation times of Brownian motion and gives a quantitative version of the fact that Brownian particles which hit a set 'stay around for some time'. This will allow us to estimate in trace norm the effect of an additional Dirichlet boundary condition where the original potential is large. As the use of occupation times already indicates, we employ the Feynman-Kac formula for this purpose.

To give a flavour of our main result, let us formulate it for the special case where $V$ has spherical barriers of radius $R_{n}$, width $w_{n}$ and height $h_{n}$. If these barriers (not necessarily concentric) divide $\mathbb{R}^{d}$ into bounded components, and if

$$
\sum_{n} R_{n}^{d-1} e^{-\eta w_{n}\left(h_{n}\right)}<\infty
$$

for some $\eta<1 / 8 \sqrt{ } 2$, then

$$
\sigma_{\mathrm{ac}}\left(-\frac{1}{2} \Delta+V\right)=\varnothing
$$

Received 22 October 1993.

1991 Mathematics Subject Classification 81Q10.

Bull. London Math. Soc. 27 (1995) 162-168 
In this summability condition there is an additional feature, which, of course, does not occur in the one-dimensional case: besides height and width there is also a dependence on the surface area of the barrier. For general barriers we measure this by the generalized area, introduced at the beginning of the following section.

Although we prove absence of absolute continuity by comparing $-\frac{1}{2} \Delta+V$ with an operator with pure point spectrum, we cannot expect this kind of spectrum for the general potentials which are covered by our main theorem. This is known from several results in one dimension: in [5] it was shown for a class of potentials on the half line $(0, \infty)$ that dense pure point spectrum occurs for almost every boundary condition at 0 . Nevertheless, a general result announced in [2] says that for some boundary conditions these potentials will yield purely continuous spectrum. Finally, a result in [3] states that for other special cases of the Simon-Spencer class, which are closely related to the examples given in [6], the spectrum is singular continuous for every boundary condition. In these examples the singular continuity will be stable even under perturbation by a compactly supported potential.

\section{The results}

We shall consider the Schrödinger operator $H=-\frac{1}{2} \Delta+V$ in $L_{2}\left(\mathbb{R}^{d}\right)$, where $V_{+} \in$ $L_{\text {loc }}^{1}\left(\mathbb{R}^{d}\right)$ and $V_{-} \in K_{d}$, the $d$-dimensional Kato class (compare [9] for the relevant definitions). In the sequel we say that the potential $V$ has barriers of form $S_{n}$, height $h_{n}$ and width $w_{n}$, if

(B.1) the $S_{n}$, for $n \in \mathbb{N}$, are compact subsets of $\mathbb{P}^{d}$ with Lebesgue measure zero and such that $\mathbb{R}^{d} \backslash \bigcup_{n \in \mathbb{N}} S_{n}$ is open and has only bounded connected components, (B.2) $V(x) \geqslant h_{n}$ if $\operatorname{dist}\left(x, S_{n}\right) \leqslant w_{n} / 2, n \in \mathbb{N}$.

We also use the generalized area $\sigma(S)$ for a compact subset $S$ of $\mathbb{R}^{d}$ :

$$
\sigma(S)=\sup _{r \geqslant 0} \frac{\operatorname{meas}\{x ; r \leqslant \operatorname{dist}(x, S) \leqslant r+1\}}{r^{d}+1} .
$$

Essentially the same definition of $\sigma(S)$ and some discussion of this notion were given in [12].

THEOREM. Assume that $V$ has barriers of form $S_{n}$, height $h_{n}$ and width $w_{n}$. If

$$
\sup _{n} \frac{w_{n}}{\sqrt{ }\left(h_{n}\right)}<\infty
$$

and

$$
\sum_{n} \sigma\left(S_{n}\right) \exp \left(-\eta w_{n} \sqrt{ }\left(h_{n}\right)\right)<\infty
$$

for some $\eta<1 / 8 \sqrt{ } 2$, then

$$
\sigma_{\mathrm{ac}}\left(-\frac{1}{2} \Delta+V\right)=\varnothing .
$$

Note that by the definition of $\sigma(S)$ we have $\sigma\left(S_{n}\right) \geqslant C>0$ uniformly in $n$. Therefore (C.2) implies $w_{n} \sqrt{ }\left(h_{n}\right) \rightarrow \infty$ and, together with (C.1), $h_{n} \rightarrow \infty$, meaning the existence of high barriers. A typical special case of (C.1) is $h_{n} \rightarrow \infty$ and $w_{n}$ bounded. In case of unbounded $w_{n}$, that is, wide barriers, the results of [12] will often apply.

Before going into the proof of the Theorem, we collect together some basic facts in three lemmas. The first gives a general trace class criterion for operators in $L_{2}$ over some $\sigma$-finite measure space. $\mathfrak{B}$ denotes the bounded operators. 
Lemma 1. Let $T \in \mathfrak{B}\left(L_{1}, L_{2}\right), S \in \mathfrak{B}\left(L_{2}, L_{1}\right)$ and $\Phi \in L_{1}$ such that

$$
|S f(x)| \leqslant \Phi(x)
$$

for a.e. $x$ and all $f \in L_{2}$ with $\|f\|_{2} \leqslant 1$. Then $T S$ is trace class with trace norm

$$
\|T S\|_{\mathrm{tr}} \leqslant\|\Phi\|_{1}\|T\| \text {. }
$$

\section{Proof. See [12, Lemma 2.1].}

In the sequel we shall use probabilistic methods, and thus we let $\left(\Omega^{x}, \mathbb{P}^{x},\left(X_{t}\right)_{t \geqslant 0}\right)$ denote Brownian motion starting in $x \in \mathbb{R}^{d}$ with expectation $\mathbb{E}^{x}$ (see [8, II.4] for background information). For $\omega \in \Omega^{x}$ and $S \subset \mathbb{R}^{d}$, the first hitting time of $S$ is given by $\tau_{S}(\omega)=\inf \{t>0 ; X,(\omega) \in S\}$.

Lemma 2. (a) Given $t>0$ and $\varepsilon>0$, there exists $C=C(d, t, \varepsilon)$ such that for all $r>0$

$$
\mathbb{P}^{0}\left[\tau_{\{y ;|y|=r\}} \leqslant t\right] \leqslant C \exp \left(-\frac{r^{2}}{(2+\varepsilon) t}\right) .
$$

(b) For $t>0$ and $p>0$, there exists $C=C(d, t, p)$ such that for all compact $S \subset \mathbb{R}^{d}$

$$
\int_{\mathbb{R}^{d}}\left(\mathbb{P}^{x}\left[\tau_{s} \leqslant t\right]\right)^{p} d x \leqslant C \sigma(S) .
$$

Proof. (a) By the reflection principle (compare [8, Theorem 3.6.5, p. 25]),

$$
\mathbb{P}^{0}\left[\tau_{\{y ;|y|-r\}} \leqslant t\right] \leqslant 2 \mathbb{P}^{0}\left[\left|X_{t}\right| \geqslant r\right] \leqslant 2(2 \pi t)^{-d / 2} \int_{|y| \geqslant r} \exp \left(-\frac{|y|^{2}}{2 t}\right) d y,
$$

which yields the required estimate.

(b) Since $\mathbb{P}^{x}\left[\tau_{s} \leqslant t\right] \leqslant \mathbb{P}^{0}\left[\tau_{\{y:|y|-\text { dist }(x, S)\}} \leqslant t\right]$, part (a) with $\varepsilon=1$ and the definition of $\sigma(S)$ imply

$$
\begin{aligned}
\int\left(\mathbb{P}^{x}\left[\tau_{s} \leqslant t\right]\right)^{p} d x & =\sum_{n \geqslant 0} \int_{n \leqslant \text { dist }(x, S) \leqslant n+1}\left(\mathbb{P}^{x}\left[\tau_{s} \leqslant t\right]\right)^{p} d x \\
& \leqslant \sum_{n \geqslant 0} \sigma(S)\left(n^{d}+1\right) C^{p} \exp \left(-\frac{n^{2} p}{3 t}\right) \\
& =C(d, t, p) \sigma(S) .
\end{aligned}
$$

In the last of our three lemmas we estimate the probability that a particle hits $S$ but spends only little time in a neighbourhood of $S$. To this end we introduce the occupation time of a $\delta$-neighbourhood,

$$
T_{S, \delta}(\omega):=\operatorname{meas}\left\{u \in(0, t) ; \operatorname{dist}\left(X_{u}(\omega), S\right) \leqslant \delta\right\} .
$$

Lemma 3. Let $t>0, \varepsilon>0$. Then there exists $C=C(d, t, \varepsilon)$ such that for all $0<\alpha<t$ and $S \subset \mathbb{B}^{d}$ compact,

$$
\mathbb{P}^{x}\left[\tau_{s} \leqslant t, T_{S . \delta} \leqslant \alpha\right] \leqslant C \exp \left(-\frac{\delta^{2}}{4(2+\varepsilon) \alpha}\right)
$$


Proof. Let $B:=\{x ; \operatorname{dist}(x, S) \leqslant \delta\}$ and $B^{\prime}:=\{x ; \operatorname{dist}(x, S) \leqslant \delta / 2\}$. Denote by $\tau^{\prime}$ the first hitting time of $B^{\prime}$, and let $T^{\prime}:=\inf \left\{s>0 ;\left|X_{s+\tau^{\prime}}-X_{\tau^{\prime}}\right| \geqslant \delta / 2\right\}$ on $\left\{\tau^{\prime}<\infty\right\}$, and infinity otherwise. We have

$$
\mathbb{P}^{x}\left[\tau_{S} \leqslant t, T_{S, \delta} \leqslant \alpha\right] \leqslant \mathbb{P}^{x}\left[\tau_{S} \leqslant t, \tau^{\prime} \leqslant t-\alpha, T_{S, \delta} \leqslant \alpha\right]+\mathbb{P}^{x}\left[\tau_{s} \leqslant t, \tau^{\prime}>t-\alpha\right] .
$$

The first summand can be estimated by

$$
\mathbb{P}^{x}\left[\tau_{s} \leqslant t, T^{\prime} \leqslant \alpha\right]
$$

since $X_{s+r^{\prime}} \in B$ as long as $\left|X_{s+\tau^{\prime}}-X_{\tau^{\prime}}\right| \leqslant \delta / 2$. The second term is zero if $x \in B^{\prime}$; for $x \notin B^{\prime}$ it follows that $\operatorname{dist}\left(X_{\tau^{\prime}}(\omega), S\right)=\delta / 2$ for $\omega \in \Omega^{x}$, so that the second term can be majorized by

Put together, this gives

$$
\mathbb{P}^{x}\left[T^{\prime} \leqslant \alpha\right]
$$

$$
\mathbb{P}^{x}\left[\tau_{S} \leqslant t, T_{S, \delta} \leqslant \alpha\right] \leqslant 2 \mathbb{P}^{x}\left[T^{\prime} \leqslant \alpha\right] .
$$

By the strong Markov property and translation invariance of Brownian motion (compare [8, Theorem 7.9, p. 68]),

$$
\mathbb{P}^{x}\left[T^{\prime} \leqslant \alpha\right] \leqslant \mathbb{P}^{0}\left[\tau_{\{y ;|y|-\delta / 2\}} \leqslant \alpha\right] \leqslant C \exp \left(-\frac{\delta^{2}}{4(2+\varepsilon) \alpha}\right),
$$

according to Lemma 2(a).

Proof of the Theorem. We define $H=-\frac{1}{2} \Delta+V$ on $L_{2}\left(\mathbb{R}^{d}\right)$ and $H_{G}=-\frac{1}{2} \Delta+V$ on $L_{2}(G)$ with Dirichlet boundary conditions, for arbitrary open $G \subset \mathbb{R}^{d}$, via the corresponding quadratic forms.

Fix $S_{n}, h_{n}, w_{n}$ as in the assumption of the Theorem, and let

$$
H_{D}:=H_{\mathbb{R}^{d} \backslash \cup_{n} s_{n}}=\underset{i}{\oplus} H_{U_{i}},
$$

where $U_{i}$ are the connected components of $\mathbb{R}^{d} \backslash \bigcup_{n} S_{n}$. Since the $U_{i}$ are bounded, $H_{U_{t}}$ has discrete spectrum and consequently $\sigma_{\mathrm{ac}}\left(H_{D}\right)=\varnothing$. Thus if we manage to construct wave operators for $H, H_{D}$, the assertion of the Theorem will be proved. By the wellknown invariance principle (see, for example, [7, Corollary 4, p. 31]), it is sufficient to show that $\exp (-2 t H)-\exp \left(-2 t H_{D}\right)$ is trace class for some $t>0$. Writing

we have that

$$
H_{0}:=H, \quad H_{n}:=H_{\mathbb{R}^{d} \backslash \bigcup_{k=1}^{n} s_{k}},
$$

$$
\exp (-2 t H)-\exp \left(-2 t H_{D}\right)=\sum_{n=1}^{\infty}\left(\exp \left(-2 t H_{n-1}\right)-\exp \left(-2 t H_{n}\right)\right)
$$

in the strong sense (by monotone form convergence). We shall now demonstrate that assumptions (C.1) and (C.2) imply that

$$
\sum_{n=1}^{\infty}\left\|\exp \left(-2 t H_{n-1}\right)-\exp \left(-2 t H_{n}\right)\right\|_{\mathrm{tr}}<\infty,
$$

which suffices for the desired trace class property. To estimate $\| \exp \left(-2 t H_{n-1}\right)-$ $\exp \left(-2 t H_{n}\right) \|_{\mathrm{tr}}$, we shall apply Lemma 1 , and therefore factorize

$$
e^{-2 t H_{n-1}}-e^{-2 t H_{n}}=e^{-t H_{n-1}}\left(e^{-t H_{n-1}}-e^{-t H_{n}}\right)+\left(e^{-t H_{n-1}}-e^{-t H_{n}}\right) e^{-t H_{n}} .
$$


By (C.2) there is an $\varepsilon>0$ such that $\sum_{n} \sigma\left(S_{n}\right) \exp \left((-1 / 8 \sqrt{ }(2+\varepsilon)) w_{n} \sqrt{ }\left(h_{n}\right)\right)<\infty$. Fix $t>\sup _{n}(1 / 8 \sqrt{ }(2+\varepsilon)) w_{n}\left(h_{n}\right)^{-1 / 2}$ which, according to assumption (C.1), is possible. In order to apply Lemma 1 , we let $f \in L_{2},\|f\|_{2} \leqslant 1$ and use the Feynman-Kac formula (compare [8, II.6]) to write

$$
\left|\left(e^{-t H_{n-1}}-e^{-t H_{n}}\right) f(x)\right|=\left|\mathbb{E}^{x}\left[\exp \left(-\int_{0}^{t} V \circ X_{s} d s\right) f \circ X_{t}, \tau_{\cup_{k=1}^{n-1} s_{k}}>t, \tau_{s_{n}} \leqslant t\right]\right| .
$$

Applying Cauchy-Schwarz twice, we obtain

$$
\begin{aligned}
\ldots & \leqslant\left(\mathbb{E}^{x}\left[\exp \left(-2 \int_{0}^{t} V_{+} \circ X_{s} d s\right), \tau_{S_{n}} \leqslant t\right]\right)^{1 / 2}\left(\mathbb{E}^{x}\left[\exp \left(2 \int_{0}^{t} V_{-} \circ X_{s} d s\right)|f|^{2} \circ X_{t}\right]\right)^{1 / 2} \\
& \leqslant(\ldots)^{1 / 2}\left\|e^{\left(-1 / 2 \Delta-2 V_{-}\right) t}: L_{1} \rightarrow L_{\infty}\right\|^{1 / 2} \\
& \leqslant C \cdot\left(\mathbb{E}^{x}\left[\exp \left(-\int_{0}^{t} 4 V_{+} \circ X_{s} d s\right), \tau_{s_{n}} \leqslant t\right]\right)^{1 / 4}\left(\mathbb{P}^{x}\left[\tau_{s_{n}} \leqslant t\right]\right)^{1 / 4} .
\end{aligned}
$$

Here we have used that the norm occurring above is finite by [9, Theorem B.1.1, p. 460 ] and, again, Feynman-Kac. Lemma 2(b) gives us control on the last term on the right. To estimate the remaining factor uniformly in $x$, we use the occupation time estimate from Lemma 3 together with the fact that $V_{n}$ is large in a $w_{n} / 2$ neighbourboood of $S_{n}$ : fix $n \in \mathbb{N}$ and let $T_{n}(\omega):=\operatorname{meas}\left\{u \in(0, t) ; \operatorname{dist}\left(X_{u}(\omega), S_{n}\right) \leqslant\right.$ $\left.w_{n} / 2\right\}$. Since $\int_{0}^{t} V_{+} \circ X_{s} d s \geqslant h_{n} T_{n}$ we have, for $0<\alpha<t$,

$$
\mathbb{E}^{x}\left[\exp \left(-\int_{0}^{t} 4 V_{+} \circ X_{s} d s\right), \tau_{s_{n}} \leqslant t\right] \leqslant e^{-4 h_{n} \alpha} \mathbb{P}^{x}\left[T_{n}>\alpha\right]+\mathbb{P}^{x}\left[\tau_{s_{n}} \leqslant t, T_{n} \leqslant \alpha\right] .
$$

Using Lemma 3 this can be estimated by

$$
\exp \left(-4 h_{n} \alpha\right)+C \exp \left(-\frac{\left(w_{n} / 2\right)^{2}}{4(2+\varepsilon) \alpha}\right)
$$

Setting $4 h_{n} \alpha=\left(w_{n} / 2\right)^{2} / 4(2+\varepsilon) \alpha$, we arrive at $\alpha=(1 / 8 \sqrt{ }(2+\varepsilon)) w_{n}\left(h_{n}\right)^{-1 / 2}<t$ by our assumption on $t$. With this choice of $\alpha$, we obtain

$$
\mathbb{E}^{x}\left[\exp \left(-\int_{0}^{t} 4 V_{+} \circ X_{s} d s\right), \tau_{s_{n}} \leqslant t\right] \leqslant C \exp \left(-\frac{w_{n} \sqrt{ }\left(h_{n}\right)}{2 \sqrt{ }(2+\varepsilon)}\right) .
$$

Plugging this into (3), we find

$$
\left|\left(e^{-t H_{n-1}}-e^{-t H_{n}}\right) f(x)\right| \leqslant C \exp \left(-\frac{w_{n} \sqrt{ }\left(h_{n}\right)}{8 \sqrt{ }(2+\varepsilon)}\right)\left(\mathbb{P}^{x}\left[\tau_{S_{n}} \leqslant t\right]\right)^{1 / 4}=: \Phi_{n}(x)
$$

for all $f \in L_{2},\|f\|_{2} \leqslant 1$. Hence we can apply Lemma 2(b) to estimate

$$
\left\|\Phi_{n}\right\|_{1} \leqslant C \cdot \exp \left(-\frac{1}{8 \sqrt{ }(2+\varepsilon)} w_{n} \sqrt{ }\left(h_{n}\right)\right) \cdot \sigma\left(S_{n}\right) .
$$

Since $\left\|e^{-t H_{n}}: L_{1} \rightarrow L_{2}\right\| \leqslant\left\|e^{-t H}: L_{1} \rightarrow L_{2}\right\|$ (monotonicity), it follows from Lemma 1 that

$$
\left\|e^{-t H_{n-1}}\left(e^{-t H_{n-1}}-e^{-t H_{n}}\right)\right\|_{\mathrm{tr}} \leqslant C \cdot \sigma\left(S_{n}\right) \exp \left(-\frac{1}{8 \sqrt{ }(2+\varepsilon)} w_{n} \sqrt{ }\left(h_{n}\right)\right)
$$


As $\left\|\left(e^{-t H_{n-1}}-e^{-t H_{n}}\right) e^{-t H_{n}}\right\|_{\mathrm{tr}}=\left\|e^{-t H_{n}}\left(e^{-t H_{n-1}}-e^{-t H_{n}}\right)\right\|_{\mathrm{tr}}$, we finally obtain

$$
\left\|e^{-2 t H_{n-1}}-e^{-2 t H_{n}}\right\|_{\mathrm{tr}} \leqslant C \sigma\left(S_{n}\right) \exp \left(-\frac{1}{8 \sqrt{ }(2+\varepsilon)} w_{n} \sqrt{ }\left(h_{n}\right)\right),
$$

which by (C.2) implies the summability of the trace norms, and hence the Theorem.

We conclude with some remarks.

(a) Assume that $V$ has barriers of form $S_{n}$, height $h_{n}$ and width $w_{n}$ with the property that for each subsequence $S_{n_{k}}$ the connected components of $\mathbb{R}^{d} / \bigcup_{k} S_{n_{k}}$ are bounded. Then (C.1) and

$$
\lim _{n} \sigma\left(S_{n}\right) \exp \left(-\eta w_{n} \sqrt{ }\left(h_{n}\right)\right)=0
$$

for some $\eta<(8 \sqrt{ } 2)^{-1}$ already implies that

$$
\sigma_{\mathrm{ac}}\left(-\frac{1}{2} \Delta+V\right)=\varnothing \text {. }
$$

To see this one has only to apply the Theorem to a suitable subsequence $S_{n_{k}}$.

(b) For $d=1$ and $S_{n}=\left\{x_{n}\right\}$ satisfying $(-1)^{k} x_{k} \rightarrow \infty$ for $k \rightarrow \infty$, we are in the situation of (a). As, moreover, $\sigma\left(S_{n}\right)$ is constant in this case, the summability condition of the Theorem can be weakened to

$$
\lim _{n} w_{n} \sqrt{ }\left(h_{n}\right)=\infty
$$

Thus we obtain as a special case of our Theorem the original result from [10]. (From $h_{n} \rightarrow \infty$ it follows that in (4) we can assume boundedness of $w_{n}$, and therefore also (C.1) is satisfied.)

(c) If the potential $V$ is spherically symmetric, then by separation of variables the one-dimensional result of [10] applies directly. This gives a slightly stronger result than our Theorem, since only $h_{n} \rightarrow \infty$ and $w_{n} \sqrt{ }\left(h_{n}\right) \rightarrow \infty$ are required, and no growth condition on the $\sigma\left(S_{n}\right)$.

(d) In order to obtain $\sigma_{\mathrm{ac}}=\varnothing$, the barriers $S_{n}$ need not be 'massive'. One may cut holes into the $S_{n}$ (thus violating property (B.1) of barriers) as long as the holes get small quickly enough as $n \rightarrow \infty$ to allow another trace class perturbation argument. More interesting would be an answer to the following question. May the holes be big as long as the hole in $S_{n}$ is far apart from that in $S_{n+1}, n=1, \ldots$ ? Think of concentric $S_{n}$ with holes in alternating directions.

\section{References}

1. J. M. COMBes and P. D. Hislop, 'Some transport and spectral properties of disordered media', Proceedings of the Workshop on Schrödinger operators, Aarhus 1991, Lecture Notes in Phys. (ed. E. Balslev, Springer, Berlin, 1992).

2. R. Del Rio, S. Jitomirskaya, M. Makarov and B. Simon, 'Singular continuous spectrum is generic', Preprint, 1993.

3. A. Y. Gordon, S. A. Molchanov and B. TSaGani, 'Spectral theory for one-dimensional Schrödinger operators with strongly fluctuating potentials', Funct. Anal. Appl. 25 (1992) 236-238.

4. P. D. Hislop and S. Nakamura, 'Stark Hamiltonians with unbounded random potentials', Rev. Math. Phys. 2 (1990) 479-494.

5. W. KIrSCh, S. A. Molchanov and L. A. Pastur, One-dimensional Schrödinger operators with high potential barriers, Oper. Theory: Adv. Appl. 57 (Birkhäuser, Basel, 1992) 163-170.

6. D. Pearson, 'Singular continuous measures in scattering theory', Comm. Math. Phys. 60 (1978) 13-36. 
7. M. ReED and B. Simon, Methods of modern mathematical physics, III. Scattering theory (Academic Press, New York, 1979).

8. B. Simon, Functional integration and quantum physics (Academic Press, New York, 1979).

9. B. Simon, 'Schrödinger semigroups', Bull. Amer. Math. Soc. 7 (1982) 447-526.

10. B. SimON and T. SPENCER, 'Trace class perturbations and the absence of absolutely continuous spectra', Comm. Math. Phys. 125 (1989) 113-125.

11. P. StollmanN, 'Scattering by obstacles of finite capacity', J. Funct. Anal. 121 (1994) 416-425.

12. P. Stollmann and G. Stolz, 'Singular spectrum for multidimensional Schrödinger operators with potential barriers', J. Operator Theory, to appear.

13. G. STOLz, 'Spectral theory for slowly oscillating potentials, II. Schrödinger operators', Math, Nachr., to appear.

14. G. Stolz, "Note to the paper by P. D. Hislop and S. Nakamura : Stark Hamiltonians with unbounded random potentials', Rev. Math. Phys. 5 (1993) 453-456.

\author{
I. McGillivray \\ Fachbereich Mathematik \\ Universität Potsdam \\ Postfach 601553 \\ D-14415 Potsdam \\ Germany
}

P. Stollmann and G. Stolz

Fachbereich Mathematik

Johann Wolfgang Goethe-Universität

D-60054 Frankfurt am Main

Germany 